\title{
Educología, emergente ciencia o epistemología de la educación
}

\author{
Por. Dr. Alejandro Rubina López \\ allicorubina@hotmail.es \\ https://orcid.org/0000-0003-1421-7043
}

Docente de Posgrado de la Facultad de Educación

de la Universidad Nacional "Hermilio Valdizán” de Huánuco

\section{RESUMEN}

La presente investigación, tuvo como objetivo explicar desde el punto de vista epistemológico, que la Educología constituye una emergente ciencia o epistemología de la educación. La educología, como ciencia emergente, tiene propio campo de estudio, está conformado por hechos y fenómenos educativos. El método utilizado fue la hermenéutica que es un método filosófico que consiste en interpretar y explicar el significado de las cosas. Los resultados, nos indican que; existen categorías propias afines a la educología, como: Educología, educólogo, educogenia, educocionístico, entre otros; que son categorías educológicas que están relacionados con la Educología que le dan personalidad propia como ciencia. En conclusión, la Educología se configura como una nueva ciencia o epistemología de la educación por tener propio objeto de estudio, por cuanto; la educación en sí misma no puede ser ciencia, tampoco la pedagogía. La educación como un hecho o fenómeno educativo constituye campo de estudio de la ciencia emergente llamado Educología.

Palabras Clave: educología; educocionístico; educólogo; epistemología. 


\title{
Educology, emerging science or epistemology of education
}

\begin{abstract}
The present research aimed to explain from the epistemological point of view, that Educology constitutes an emerging science or epistemology of education. Educology, as an emerging science, has its own field of study, it is made up of educational facts and phenomena. The method used was hermeneutics, which is a philosophical method that consists of interpreting and explaining the meaning of things. The results indicate that; There are own categories related to educology, such as: Educology, educologist, educogeny, educocionist, among others; which are educational categories that are related to Educology that give it its own personality as a science. In conclusion, Educology is configured as a new science or epistemology of education because it has its own object of study, since; education in itself cannot be science, nor can pedagogy. Education as an educational fact or phenomenon constitutes an emerging field of study called Educology.
\end{abstract}

Key Words: Educology, educationist, educologist, epistemology.

Artículo recibido: 20 diciembre. 2021 Aceptado para publicación: 10 enero 2022 Correspondencia: allicorubina@hotmail.es Conflictos de Interés: Ninguna que declarar 


\section{INTRODUCCIÓN}

En el presente trabajo de investigación, se analiza sobre la lucha epistemológica entre el denominado educación y pedagogía que se disputan la categoría de ser ciencia.

También el presente trabajo tiene como objetivo demostrar mediante argumentos epistemológicos que la Educología, constituye una ciencia emergente que tiene como objeto o campo de estudio a la educación, por cuanto; la educación resulta ser objeto de estudio o campo de conocimiento de la Educología.

La educación constituye un hecho, proceso y fenómeno educativo que requiere ser estudiada por una ciencia llamada Educología.

En este contexto, la Pedagogía no es una ciencia, sino una disciplina de la ciencia educológica, por cuanto, la Pedagogía no teoriza, sino que es una aplicación práctica referido a la aplicación de la didáctica que es indispensable en el proceso de enseñanza aprendizaje.

Tampoco la educación puede configurarse como ciencia, por cuanto resultar ser un hecho o fenómeno educativo.

Nuestra inquietud para investigar sobre el dilema de la existencia de la lucha y disputa epistemológica, entre la Educación y la Pedagogía surge cuando por un lado existen estudiosos que señalan en decir que educación es ciencia.

Por otro lado, también hay investigadores que defienden en decir que la pedagogía es ciencia y un tercer grupo afirman que ambos, tanto la educación y pedagogía es ciencia. Estas tres posturas, ya representa una divergencia, controversia y una lucha epistemológica que se disputan de un objeto o campo de estudio, ¿Cuál es ese campo de estudio que se disputan? Se disputan a la educación como un hecho o fenómeno que resulta ser un campo de estudio de otra ciencia emergente llamado educología.

En este contexto, el objetivo de la presente investigación es fundamentar desde el punto de vista epistemológico que la educación no constituye ciencia, ni la pedagogía puede llamarse ciencia, por cuanto; el primero constituye un hecho, proceso y fenómeno social que requiere ser estudiada por una determinada ciencia, por consiguiente, no puede llamarse ciencia la educación.

Igualmente, la pedagogía no se configura como ciencia, por cuanto; la pedagogía no teoriza sobre la educación por constituir una praxis de la educación. 
Tampoco la educación y pedagogía pueden ser ciencia ambos al mismo tiempo, me pregunto: ¿cuál sería su campo de estudio?

La ciencia que estudia el hecho o fenómeno educativo constituye la Educología, por cuanto; tiene una estructura teórica y metodológica para denominarse ciencia.

Existen importantes estudios y ensayos publicados en importantes revistas electrónicas que defienden a la educación y Pedagogía como ciencia.

Dentro de la vieja lucha epistemológica hay destacados tratadistas que afirman en decir que la EDUCACIÓN es ciencia y otros que niegan a la educación como ciencia

Entre los autores que sostienen en decir que la educación no es ciencia, sino más bien afirman en decir que la educación constituye objeto del conocimiento.

Bárcena, F. (2013) en su artículo titulado: Filosofía de la Educación: un aprendizaje, afirma que la educación NO puede ser considerada ciencia, por cuanto; constituye un objeto de conocimiento, al respecto explica:

"La educación, enuncia un saber y, al mismo tiempo, la experiencia de una actividad orientada a la transformación del individuo y la sociedad a través de las generaciones. La educación, entonces, es la experiencia de un encuentro (o relación) y una transmisión de la cultura (la durabilidad del mundo) entre generaciones en la filiación (y la discontinuidad) del tiempo. Como saber referido al estudio de la educación, en este sentido entendida, la filosofía de la educación posee una dimensión teórica - aspira a la elaboración"

Vega Román, E. A. (2018) en su artículo titulado: ¿Pedagogía o ciencias de la educación? una lucha epistemológica, sostiene que la educación y la pedagogía, se disputan la primacía de ser ciencia, dicha disputa o lucha desde el punto de vista epistemológico continua y es de nunca acabar, al respecto, explica:

“La pedagogía y las Ciencias de la Educación están en una disputa epistemológica ¿A quién le pertenece el campo de acción educativo? Desde hace muchos años la pedagogía no ha sido validada como la episteme que subyace los fundamentos educativos al no considerársele una ciencia, al tener un campo de acción más bien "práctico" dando cabida a la cientificidad de "las ciencias de la Educación". En el presente ensayo se lleva a cabo un análisis reflexivo sobre las diversas aristas que involucran esta discusión epistémica evidenciando y revalidando a la pedagogía como la ciencia de la educación”. 
Por nuestra parte, no comparatismo con ninguna de esas posturas, por cuanto, para nosotros la ciencia que estudia el hecho o fenómeno educativo es la Educología que tiene una estructura y un estatuto propio, por lo tanto, tiene una identidad propia.

\section{MÉTODO}

El método utilizado fue el método filosófico de la hermenéutica, que consistió en interpretar y analizar la fuente bibliográfica relacionado con las diversas propuestas teóricas sobre las controversias, divergencias y sobre la disputa o lucha epistemológica entre la educación y pedagogía que se disputan en llamarse ciencia.

Otro método usado fue el documental o análisis bibliográfico que consistió en analizar diversos artículos científicos publicados en importantes revistas indexadas de destacados autores o tratadistas que se ocupan en defender la tesis de la educación como ciencia, así como también los que defienden a la pedagogía como ciencia.

López Noguero, F. (2002) en su artículo titulado: El análisis de contenido como método de investigación, afirma que el análisis es un método que se utiliza en las investigaciones científicas, al respecto, explica:

“El vocablo «análisis» proviene del griego «analusis» (disolución) derivada, a su vez, de «analuein» (desatar, soltar).Por su parte, el Diccionario de la Real Academia Española (edición de 1992) define el término «análisis» primeramente como «distinción y separación de las partes de un todo hasta llegar a conocer sus principios o elementos», posteriormente, y en su segunda acepción encontramos que es el «examen que se hace de una obra, de un escrito o de cualquier realidad susceptible de estudio intelectual»

\section{RESULTADOS}

Como resultado de la presente investigación tenemos que existen una lucha epistemológica irreconciliables entre Educación y Pedagogía que se disputan en llamarse ciencia, aún con las dos no reúnen los requisitos para configurarse como ciencia. Dichas

\section{divergencias son los siguientes:}

1. Los que defiende a la educación como ciencia.

2. Los que abogan en defender a la pedagogía como ciencia.

3. Los que defienden tanto a la educación y pedagogía como ciencia

4. La propuesta de la Educología como una ciencia emergente o nueva epistemología de la educación 
A continuación, pasaremos a desarrollar cada uno de los argumentos del debate y lucha epistemológica entre esas tres corrientes de pensamiento filosófico epistemológico sobre la primacía de la ciencia entre la Educación y la Pedagogía.

También presentamos nuestros argumentos filosóficos y epistemológicos sobre nuestra propuesta de la Educología como una ciencia emergente ciencia o nueva epistemología de la educación.

\section{Los que defienden la educación como ciencia}

Los que defienden a la educación como ciencia, entre otros tenemos a los autores siguientes:

\section{Vicente, M.E. (2016) en su artículo titulada:}

Ciencias de la Educación: nuevas definiciones profesionales desde la historia reciente, explica: "La formación se convirtió en tarea de especialistas y profesionales, naciendo junto a ello un saber muy específico: la pedagogía (el saber enseñar), objeto de estudio de las Ciencias de la Educación".

Moreno Castillo R. (2009) en su artículo titulado: ¿Es la pedagogía una ciencia?, explica que la pedagogía no es una ciencia, al respecto explica:

En este artículo se intenta demostrar que la pedagogía no es una ciencia, sino que es un lenguaje sin significado, un discurso vacío. Los argumentos que apoyan esta tesis son fundamentalmente cuatro. El primero, la resistencia de la pedagogía a usar el método científico, niegan los hechos buscan las causas en circunstancias sociales. El segundo, la multiplicación de palabras, dando nuevos nombres a cosas que ya tenían, para así ocultar la falta de ideas bajo la capa supuestamente científica. El tercero, en la utilización de un lenguaje vacío".

Loreto Mora-Olate, M. (2020) en su investigación titulada: Educación como disciplina y como objeto de estudio: aportes para un debate, al referirse que continua el debate sobre el carácter o rango de ciencia que se la al termino educación, al respecto sostiene:

Persistir acerca del rango científico de la educación, o si es más correcto hablar de «ciencia» o «ciencias de la educación». Esta situación también conlleva implicancias en la forma en que se entiende la investigación en el campo de la educación. Al mismo tiempo, la lectura de diversos autores remite a la necesidad de buscar la precisión conceptual que conlleva a una reflexión de dichos conceptos. Es posible 
reconocer un campo denominado educación y resulta legítimo que existan diferentes saberes en ese campo, pero hay una disciplina propia llamada educación, cuyo objeto de estudio lo constituye el acto universal de enseñar, cuyo objeto de estudio es la educación".

Mateos Montero, J. (2014) en su libro titulado: Pedagogía: ¿arte o ciencia? Un viejo debate inacabado, explica que el debate sobre si la pedagogía es una ciencia o un arte, es un debate muy viejo.

\section{Los que defiende a la pedagogía como ciencia}

Entre los defensores de la Pedagogía como ciencia existen muchos estudiosos tanto a nivel nacional e internacional. Entre ellos tenemos:

Di Caudo, M. V. (2013) en su artículo titulado: La ciencia pedagógica: construcciones, disputas, desafíos. Afirma en decir que la pedagogía es ciencia, explica que la Pedagogía es la ciencia que estudia el problema de la educación en todos sus aspectos, al respecto, explica:

A lo largo de su desarrollo hubo mucha disputa en si la pedagogía era una ciencia o una técnica. Ciertamente es complejo definirla si la comparamos con otras ciencias que tienen un campo delimitado y una autonomía indiscutida (por ejemplo, las ciencias naturales o las ciencias matemáticas y otras ciencias que se denominan "duras"). La complejidad de la ciencia que nos atañe reside justamente en el hecho humano de la educación.

\section{Mendoza, C. (2004) en su artículo titulado:}

La pedagogía como ciencia: notas para un debate, al asumir la defensa de la pedagogía como ciencia, afirma diciendo que el propósito del escrito es sumarse al debate acerca del estatuto científico de la Pedagogía. Al asumir la defensa de la pedagogía como ciencia, explica:

La reflexión en torno al estatuto científico de la Pedagogía resulta obligada para quienes dedican buena parte de su quehacer a la investigación educativa; esto es, si ese saber pedagógico que se deriva de la práctica indagatoria y de aquel que se reconoce como fundamento de la profesionalidad docente, conforman un campo que define a la Pedagogía como ciencia. Un primer intento de análisis remitiría a los 
criterios que a su vez definen la constitución de los campos disciplinares, entre otros los que tienen que ver con la definición del objeto de estudio de manera clara e inequívoca, así como la determinación de los conceptos básicos y problemática que le conciernen exclusivamente de la(s) metodología(s).

\section{Los que defienden tanto a la educación y pedagogía como ciencia}

Hay una tercera corriente de opinión que considera tanto a la educación y a la pedagogía, es decir; según esta tercera postura, ambas son ciencias, opinión con la que no compartimos.

Según Vega Román, E. A (2016), hoy en día, existen tres posturas muy claras, que dependen de la escuela o tradición a la cual se adscriba, al respecto explica:

Según Ríos (2011), las tradiciones son las siguientes: Tradición alemana: La pedagogía es considerada la Ciencia de la Educación. Su origen deriva de los textos escolares didácticos de Comenio y Ranke. La pedagogía se entendió como una ciencia desde la distinción de Dilthey entre ciencias del espíritu y ciencias naturales. Siguiendo a Wulf (1999), la pedagogía en Alemania se consolida como una disciplina científica a partir de la convergencia de la pedagogía humanista, la ciencia empirista de la educación y la ciencia crítica de la misma. Tradición francesa: Las Ciencias de la Educación. El concepto de las ciencias de la educación surge bajo el contexto de normativizar y regular porciones de la recién consolidad República del Siglo XVIII, derivado de la Revolución francesa. Su consolidación llegaría más tarde, a principios del Siglo XX, basándose en un modelo de observación y cuantificación científica del "hecho educativo" o de la "educación". Dado que son un conjunto de disciplinas, las cuales tienen el mismo objetivo de estudio (educación), la pedagogía y la didáctica se convierten en instrumentos operativos de las disciplinas que operan en las ciencias de la educación. Tradición anglosajona-estadounidense. Teoría de la educación. Esta tradición tiene su origen en las teorías curriculares, en el currículo, focalizándose en lo operativo y en lo metódico. La tradición anglosajona une bajo el nombre de educación, 
la práctica y la disciplina que la estudia, concibiendo la necesidad de un discurso teórico que al mismo tiempo una apuesta práctica.

Para nuestro punto de vista no puede haber dos o más ciencias que se disputan un mismo objeto, campo de estudio o parcela del saber. Cada ciencia desde el punto de vista epistemológico campo de estudio.

Cuando dos o más ciencias, se disputan un mismo campo u objeto de estudio, pasa a constituirse como una disciplina científica, o en todo caso puede constituirse como una seudo ciencia o falsa ciencia.

Frente a tales discrepancias, consideramos que tanto la llamada ciencia de la educación y la pedagogía no constituyen ciencias.

La primera, es decir; la excusación no se configura ciencia, por cuanto; constituye un hecho, fenómeno o proceso que debe ser estudiada por una emergente ciencia llamada.

\section{EDUCOLOGÍA}

De igual manera la pedagogía no se configura como ciencia, por cuanto; no teoriza sobre la educación, la pedagogía es aplicación práctica que se constituye como una disciplina científica, en este caso se constituye como una disciplina de la Educología.

Tampoco no pueden configurarse como ciencias ambas, es decir; no pueden ser ciencia tanto la educación y la pedagogía a la vez.

La dijimos líneas más arriba, que cada saber humano, para llamarse ciencia o configurarse como ciencia debe tener entre otros su propio campo de estudio.

María Loreto Mora-Olate (2020) Educación como disciplina y como objeto de estudio: aportes para un debate, explica diciendo que la Educación, no es ciencia, sino una disciplina, al respecto, explica:

Es posible reconocer un campo denominado educación y resulta legítimo que existan diferentes saberes en ese campo, pero hay una disciplina propia llamada educación, cuyo objeto de estudio lo constituye el acto universal de enseñar, la pedagogía, que en sí entraña una complejidad por la cual confluyen diversos saberes, como la biología, la psicología, la sociología, la antropología, la filosofía, entre otros. Rojas (2006) apunta al tratamiento conceptual de la pedagogía en su movimiento entre dos tratamientos, «por una parte el estatuto de 
ciencia unificadora, y por otra el de una disciplina más dentro de un campo de conocimiento cuyo objeto de estudio es la educación»

Este autor sugiere que la educación es disciplina y al mismo tiempo objeto de estudio y también a la pedagogía lo considera como una ciencia unificadora, punto de vista con la que no compartimos.

Para nuestro punto de vista, la ciencia que estudia la educación constituye la Educología, una emergente ciencia o nueva epistemología de la educación.

\section{Propuesta de la educología como emergente ciencia o epistemología de la educación}

Nuestra propuesta sobre la Educología como emergente ciencia o epistemología de la educación, se sustenta en los fundamentos teóricos y antecedentes históricos siguientes: Los primeros académicos, estudiosos del quehacer educativo que empezaron a usar por primera vez, el termino Educología para referirse a la nueva ciencia de la educación o emergente epistemología de la educación, fueron dos destacados maestros universitarios que, en sus clases de enseñanza universitaria, tanto en pre grado y posgrado utilizaban el termino educología para referirse a la naciente ciencia de la educación o emergente epistemología educativa, llamada Educología.

Uno de los primeros maestros que frecuentemente utilizaba el término de Educología como una nueva ciencia de la educación fue el Dr. Julio Armando Ruíz Vásquez, docente de la Universidad Nacional Hermilio Valdizán de Huánuco, con quien tuve el honor de compartir lazos de amistad, por cuanto en primer lugar fue mi asesor de tesis de pre grado en la Facultad de Educación de la UNHEVAL y en segundo lugar ambos ocupamos cargos edilicios en la Comuna del Distrito de Amarilis. Fuimos autoridades edilicias del nuevo distrito de Amarilis entre los años de 1983 y 1986. El Dr. Armando Ruiz Vásquez fue el primer Alcalde del distrito de Amarilis y el autor de este artículo fue el primer Teniente de la Comuna del distrito de Amarilis.

El Dr. Julio Armando Ruíz Vásquez, por aquellos años (entre 1984 y 1985) me mostró un manuscrito de un libro acabado sobre su propuesta de dar nacimiento a una nueva epistemología o nueva ciencia de la educación llamado Educología.

El libro en manuscrito, lamentablemente no dio luz, por cuanto el Dr. Julio Armando Ruíz Vásquez murió repentinamente por haber sido asesinado, quedando truncado hasta ahora su publicación. 
Según Julio Armando Ruiz Vásquez, la educación no puede constituir en sí misma una ciencia, por cuanto; la educación se configura como un hecho o fenómeno que debe ser estudiada por otra ciencia que en este caso esa epistemología emergente se llamaría Educología.

Para nuestro punto de vista, desde la perspectiva epistemológica, cada ciencia debe tener su propio campo u objeto de estudio. La llamada ciencia de la educación no tiene su propio campo u objeto de estudio, en consecuencia, no puede configurarse como ciencia. Igualmente, la Pedagogía no puede configurarse como ciencia, por cuanto; constituye una técnica o arte de enseñar. Además, no tiene un campo de estudio propio.

Tanto la educación y la pedagogía se vienen disputando como objeto de estudio al hecho y fenómeno educativo. Es algo así como si Juan y Pedro se disputaran el amor de María. La educación en sí misma, no puede configurarse como ciencia, por cuanto la educación como un hecho o fenómeno constituye un objeto o campo de estudio de otra ciencia llamado Educología.

Otro destacado académico, que empezó usar el término Educología fue el destacado maestro universitario cantuteño el Dr. Vladimiro Guevara, de quien tuve el honor de ser su alumno en la Escuela de Posgrado de la UNHEVAL.

Vladimiro Guevara Gálvez, en sus clases siempre decía que la verdadera ciencia de la educación, es la Educología. Afirmaba que la emergente ciencia o epistemología de la educación, constituye la Educología que tiene como objeto o campo de estudio el hecho o fenómeno educativo.

Igualmente negaba que la pedagogía fuese ciencia, por cuanto; constituye una técnica didáctica y arte de enseñar.

Estas dos personalidades motivaron para realizar investigación y escribir el presente artículos y reafirmar en efecto, que ni la educación ni la pedagogía pueden configurarse como ciencia, por cuanto; ningunos tienen un campo u objeto de estudio propio que es un requisito necesario e imprescindible para tener categoría de ciencia.

\section{Origen etimológico de educología}

Educología, es un término compuesto conformado por dos voces o vocablos conformados por:

- Educo que significa educar, dar o brindar educación

- Logos o logia que significa estudio o tratado. 
Resumiendo, y juntando ambos vocablos en un solo término compuesto, resulta Educología.

\section{¿Qué es educología?}

Definiremos a la educología como la teoría de la educación, es decir, la educología es la ciencia o epistemología que tiene como objeto de estudio o campo investigativo, el hecho o fenómeno educativo.

Todo saber humano para alcanzar el grado o título de ciencia debe tener una estructura epistémica que le dan personalidad propia. Uno de los requisitos para llamarse ciencia está referido al objeto o campo de estudio propio.

La educología cumple con ese requisito, no necesita vientre de alquiler para pretender configurarse como ciencia. Como insistimos el objeto o campo de estudio propio de la educología está conformado por el hecho o fenómeno educativo desde una visión holística considerando como parte de la educación a la madre naturaleza o medio ambiente que nos rodea. También la Educología como ciencia tiene sus propias categorías epistemológicas que le dan personalidad propia como ciencia autónoma o nueva epistemología de la ciencia.

\section{Entre las principales categorías o palabras clave tenemos lo siguiente.}

1. Educología

2. Educólogo

3. Educogenia

4. Educocionístico

5. Educando

6. Educere

7. Educo

La Educología. como una ciencia emergente tiene también sus ramas, está conformado por la metodología educológica, epistemología educogenia, educología cognitiva, educología ecológica, educología digital, entre otros.

\section{Estructura de la ciencia}

Para llamarse ciencia. debe tener una estructura conformado por diversos elementos que le permite tener una identidad propia.

Es como una casa, para llamarse casa debe tener una estructura sólida, de lo contrario se cae. 
Así lo mismo una ciencia. para llamarse como tal, debe tener una estructura propia.

Toda ciencia debe tener un objeto de estudio, métodos, teoría, problemas hipótesis, leyes, ramas, disciplinas entre otros.

Todo saber humano, para llamarse o configurarse como ciencia debe tener una estructura. Esa estructura, cual las columnas de un edificio le dan solidez teórica epistemológica para llamarse ciencia, de lo contrario se convertiría en una falsa o seuda ciencia, o en todo caso, o simplemente se convertiría solo como en una disciplina científica.

Viñas, A. (1960) en su libro titulado: La interpretación estructural en la ciencia moderna: aportaciones para una teoría de la estructura económica, sostiene que el concepto de "estructura" se define corrientemente en relación con el de "sistemas", al respecto explica: Cuando se habla de "estructura" es difícil evitar la connotación de que tal concepto se aplica también a "sistema", en tanto que cuando se habla de éstos la idea inmediata es la de totalidades estructuradas de elementos. En un intento previo podríamos, pues, aproximarnos a la noción de estructura de un sistema indicando que es la parte relativamente más permanente de las relaciones existentes entre los componentes que lo integran como totalidad (superior) en tanto que, como afirma Jordán, "denominamos sistema a una cosa cuando queremos expresar el hecho de que la cosa se percibe como compuesta de un conjunto de elementos, o partes, interconectados entre sí en virtud de un principio discernible, distinguible.

En efecto cuando nos referimos a la a estructura de la ciencia, nos estamos refiriendo que la ciencia es un sistema de conocimientos que tienen un conjunto de elementos o características, que le permiten configurarse a una determinada como una ciencia. A esos elementos o características, se denomina estructura de la ciencia

\section{Estos elementos estructurales de la ciencia están conformados por:}

1. Objeto de estudio

2. Métodos

3. Problemas

4. Categorías

5. Hipótesis

6. Teoría

7. Leyes

8. Ramas 
9. Disciplinas

\section{Estructura de la ciencia}

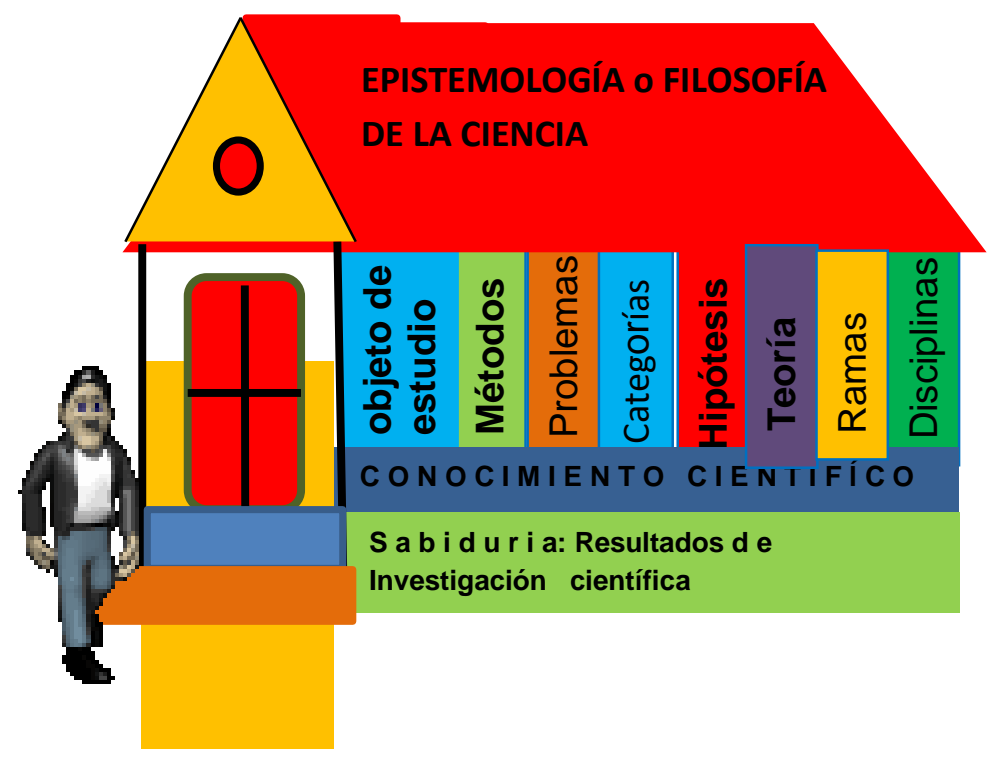

\section{Objeto de estudio}

Cualquier disciplina científica que aspira a convertirse o quiere configurarse como una ciencia autónoma debe tener su propio objeto o campo de estudio.

Una ciencia sin un campo de estudio propio, no sería ciencia, seria disciplina o ciencia en formación, o en todo caso sería una seuda ciencia o falsa ciencia.

Desde este punto de vista, también una investigación científica tiene su objeto de estudio, dicho objeto de estudio se refiere al problema que se investiga.

Meyer Rodríguez, J.A. (2010) en su artículo titulado: El objeto de estudio como sustento esencial de la investigación en Comunicación, señala que el objeto de estudio se refiere al problema o fenómeno de estudio dentro de una ciencia o investigación científica, al respecto, explica:

En tal forma, delimitar objetos de estudio no es repetir mecánicamente algunos pasos de carácter metodológico sino abordar un problema o fenómeno a partir de textos teóricos referentes con el propósito de contribuir al avance de una línea de investigación en esta área del conocimiento. La delimitación del objeto supone también una inquietud por parte del investigador que lo lleva a definir una cierta temática de interés e invertir recursos para tratar de aprehenderla. 
Educología, emergente ciencia o epistemología...

\section{MÉTODOS}

Toda ciencia debe tener sus propios métodos, técnicas e instrumentos de investigación científica.

Aun cuando existen métodos generales que son válidos para todas las ciencias, cada ciencia debe tener sus propios métodos, técnicas e instrumentos de investigación.

Morales, V. (2002) en su artículo titulado: Sobre la metodología como ciencia y el método científico: un espacio polémico, sostiene que existen numerosas metodologías: de investigación, de enseñanza, de planeamiento, de producción industrial o tecnológica, al respecto explica:

El estudio de la problemática de los métodos compete a una disciplina antigua muy importante, poco sistematizada, muy relacionada con la lógica formal y con bibliografía escasa, denominada Metodología General o, simplemente, Metodología, término que tiene dos acepciones o significados usuales, de los cuales acogemos el primero, porque es el principal y objeto de la presente exposición. La acepción amplia (y etimológica), según la cual, como dijimos, Metodología es la ciencia de los métodos, es decir, es el estudio crítico del conjunto de operaciones y procedimientos racionales y sistemáticos que utiliza el ser humano para encontrar soluciones óptimas a problemas complejos, teóricos o prácticos".

Por su parte, Aguilera Hintelholher, R, M. (2013) en su artículo titulado: Identidad y diferenciación entre Método y Metodología, explica:

Los métodos de investigación pueden valorarse como un conjunto de procedimientos ordenados que permiten orientar la agudeza de la mente para descubrir y explicar una verdad. Su utilidad consiste en que tienden al orden para convertir un tema en un problema de investigación y llevar a cabo la aprehensión de la realidad. La importancia del método consiste en que está dotado de propiedades cognoscitivas que permiten el abordaje ordenado de una parte de la realidad y que depende del sujeto cognoscente la utilidad que pueda tener al conseguir que, a través del trabajo de investigación, es posible esclarecer lo que antes no se conocía. La aplicación del método no depende de sí mismo, sino del 
sujeto cognoscente, el cual con su libertad valorativa elige el objeto de estudio a investigar, selecciona el sistema de conceptos a trabajar y estructura el modo en que habrá de llevar a cabo la investigación”.

\section{TEORÍAS}

Toda ciencia tiene propias teorías. No hay ciencia sin teoría. La ciencia es teórica y práctica a la vez.

Una teoría es la explicación de la causa efecto de un problema y es la explicación racional del porqué de las cosas.

González, Rush (2006) En su ensayo titulado: Estructura, historia y unidad de la ciencia a partir de la óptica de Eduardo Nicol, sostiene que la historia de la ciencia está tejida por la secuencia de la interacción entre distintas teorías, al respecto afirma:

La evolución de la ciencia está tejida por un complejo entramado de teorías que se suceden en el tiempo. “¿Hay algún ordenamiento interno, alguna ley que regule la evolución de los sistemas?” (Nicol, 1990: 233), ¿cómo se explica la sucesión de una teoría a otra?, ¿cómo se explica, por ejemplo, el tránsito de una visión geocéntrica del universo, la heliocéntrica?

Desde este punto de vista es posible afirmar que la ciencia tiene una estructura.

\section{Objetivos}

Toda ciencia tiene determinados objetivos que se plantea. Los objetivos de la ciencia es buscar la posible verdad, lo cual se consigue mediante la investigación científica.

García, P.; Lazzari; L. y Pérez. R, (19998) en su investigación titulada: Objetivo de la ciencia, verdad y medidas de incertidumbre, sostiene que la meta de la ciencia es la verdad, respecto explica:

La meta de la ciencia es la verdad, Esta afirmación puede, en principio, parecer trivial. Sin embargo, es una afirmación no sólo discutible sino, de hecho, fuertemente discutida por importantes epistemólogos, entre los cuales tal vez el más conocido sea Larry Laudan, quien ha sostenido con insistencia que tanto la verdad como el acercamiento a la verdad (Popper habla de verosimilitud) son metas que apuntan a un "utopismo epistemológico".

González Arias, C. (2011) en su artículo titulado: La formulación de los objetivos en artículos de investigación científica en cuatro disciplinas: historia, lingüística, literatura 
y biología, sostiene que los objetivos de una investigación guían el proceso de investigación, al respecto explica:

No cabe duda que los objetivos de investigación guían el proceso de investigación y se constituyen en un elemento central de ese proceso; por lo tanto, se espera que cualquier reporte de una investigación considere los objetivos de investigación al momento de publicar los resultados, anunciados como objetivos, propiamente tal, pero también como propósitos, metas o fines de la investigación reportada. Igualmente, vale destacar que, desde un punto de vista retórico, la claridad de los objetivos y la presencia de la adecuada ubicación en un artículo también permitirían focalizar la atención del lector, facilitándole la comprensión de dicho artículo".

Desde este punto de vista, la Educología como cualquier otra ciencia, tiene sus objetivos y metas que lograr y alcanzar.

El objetivo de la educología, consisten en entrelazar lo cognitivo con la ecología.

\section{Problemas de la ciencia de la ciencia}

Toda ciencia tiene sus propios problemas que investigar, así la educología como una ciencia emergente tiene su propia problemática de estudio.

La educología se encarda de investigar, analizar, interpretar, reflexionar, sistematizar y sintetizar sobre los problemas educativos que están conformado por un conjunto de hechos, fenómenos y procesos que ocurren en un determinado contexto geográfico, medio ambiental y contexto histórico social.

DE LA SITUACIÓN PROBLEMÁTICA AL PROBLEMA Aguirre Chávez, F. (2012) en su artículo científico titulado: De la situación problemática al problema científico educacional, señala que los problemas científicos de las investigaciones educacionales emergen del conocimiento del estado actual de desarrollo del tema que se pretende investigar, al referirse al problema, explica:

El significado etimológico de la palabra problema proviene del griego probhma, próblema, «lo puesto delante», del verbo proballo, «poner delante». En el sentido primigenio, el problema emerge, se manifiesta como obstáculo que podrían tener múltiples manifestaciones: dificultades, dudas, disgustos, preocupaciones, vacíos 
cognoscitivos, anomalías, conflictos cognitivos o contradicciones. En suma, la situación problemática constituye factores o circunstancias contradictorias de vacíos cognoscitivos o de obstáculo con existencia real e independencia del sujeto; pero con afección por el que se interesa en comprenderlo y, eventualmente, resolverlo.

Desde este punto de vista todas las ciencias, tienen problemas propios que investigar.

\section{Problematización de la ciencia}

Toda ciencia se problematiza, es decir; se plantea interrogantes sobre el porqué de las cosas.

La ciencia, el científico y el investigador debe reflexionar permanentemente, es decir, en

el proceso de una investigación debe preguntarse constantemente y dar respuesta tentativa a una serie de cuestionamientos que van surgiendo en un proceso investigativo.

Sheila M. Galindo Delgados, SH. M Norma Molina Prendes, N., Moreno Campdesuñer, I. (2013) La problematización en la investigación científica. Revista Varela, Vol. 1Nro33-2012. Septiembre - diciembre. ISSN 1810-3413, al referirse a la problematización científica, afirma que la problematización va más allá del planteamiento del problema de investigación, al respeto sostiene:

Aunque es importante que el investigador aprenda a formular un problema científico, identificando las contradicciones y carencias que lo sustentan en el orden teórico y práctico, no por ello debe ser entendida la problematización como el proceso de predeterminación del problema científico y la formulación del mismo, en tal sentido se coincide con el criterio que expresa que, la problematización es un proceso que no se reduce al momento de plantear el problema; una cosa es plantear el problema y otra es resolver el problema mediante su tratamiento, transformación y solución en el proceso investigativo.

Ibarra Flor de Ma. (2016) en su investigación titulada: Importancia del Proceso de Problematización en todo Proyecto de Investigación al referirse a la problematización señala que la problematización es una elaboración que orienta la investigación, es un proceso intelectual ya que se ponen a prueba los conocimientos disciplinares adquiridos y expresa nuestras experiencias en el campo educativo, al respecto explica: 
Plantear el problema de investigación, es un proceso de recopilación y organización de información que sitúa al investigador en un contexto y orienta en la resolución de lo expuesto, por lo que se proyectan posibles propuestas de mejora. Así, la investigación educativa desde una mirada cualitativa, favorece la interrelación de saberes".

Desde este punto de vista, la Educología como ciencia se problematiza sobre el quehacer educativo, ese proceso de reflexión de carácter filosófico.

La ciencia, el científico y el investigador, en este caso el educólogo reflexiona permanentemente, es decir se pregunta del porqué de las cosas.

La problematización científica se refiere a que, en el proceso de una investigación científica, no solo se formula o plantea preguntas o interrogantes, sino que toda investigación, empieza en una problematización y termina en una problematización, es decir; en la etapa de la delimitación del problema empieza con la problematización, en el proceso de descripción o fundamentación de la investigación también debe problematizarse, es decir; el científico o investigador en todo el trayecto de un proceso de investigación no deja de problematizarse, lo que significa que hay una permanente reflexión de carácter filosófico, por cuanto la filosofía nos permite reflexionar permanentemente sobre el porqué y para que de las cosas.

\section{Ramas y disciplinas de la educología}

Toda ciencia que se configura como tal tiene ramas y disciplinas científicas.

Cuando una ciencia cuanto más se desarrolla, tiende a extenderse echando más ramas y disciplinas

\section{Entre las ramas de a educología tenemos:}

1. La pedagogía

2. Metodología educológica

3. Gnoseología educológica

4. Epistemología educológica

5. Axiología educológica

6. Ética educológica

7. Antropología educológica.

8. Sociología educológica

9. Filosofía educológica 
10. Educología ecológica

\section{Fundamentación teórica}

Al revisar la fuente bibliográfica en las bibliotecas virtuales nos damos con la sorpresa que hay muy escasa bibliografía sobre la educología, en verdad hay muy pocas investigaciones sobre esta nueva epistemología, sobre esta nueva ciencia emergente.

En la Universidad Nacional de Costa Rica, existe una división o unidad académica llamado Educología.

La división de Educología, es una de las Unidades Académicas del Centro de Investigación y Docencia en Educación (C.I.D.E.) de la Universidad Nacional, la cual brinda la formación pedagógica a los futuros profesores para la educación media principalmente, en las áreas de: Ciencias, Matemática, Español, Estudios sociales, Inglés, Francés, Educación musical, Religión, Informática educativa, Educación Comercial, Filosofía, Educación física, Ciencias agrarias y Artes plásticas en la modalidad de carreras compartidas, al respecto, explica:

La Universidad de Costa Rica, explica sobre la Unidad de Educología que tiene dicha Universidad, al respecto explica: "Es importante mencionar que contamos con una Licenciatura en Pedagogía con énfasis en didáctica y un programa de postgrado que ofrece la Maestría en Educación con mención en: docencia universitaria, atención de asuntos estudiantiles, enseñanza del Inglés como segunda lengua y enseñanza del español como segunda lengua.

Además, se cuenta con proyectos tanto en investigación como de extensión e integrados que sirven de insumo para enriquecer los diferentes procesos y dinámicas del quehacer de esta Unidad. Deseamos que encuentre la información pertinente acerca de nuestra razón de ser de acuerdo a nuestras actividades y propósitos".

Según esta información dicha universidad vienen utilizando el termino Educología, para referirse a la Educación que muchas universidades llaman ciencias de la Educación como es el caso de la Universidad Nacional "Hermilio Valdizán” de Huánuco.

Otra información que encontramos en el internet fue en una página virtual denominado EDUCOhttps://www.cide-educologia.una.ac.cr/, que lleva por título: La EDUCOLOGÍA, una nueva herramienta para el conocimiento.

En esta página, le da una definición holística a la educología, es decir; la educología es definida como ciencia que estudia el hecho y fenómeno educativo desde el punto de vista 
holístico, considerando a la educación vinculado o articulado a la ecología, al respecto al preguntarse sobre ¿Qué es la educología?, se responde y sostiene que, la educología es una nueva ciencia que fusiona la cultura ecológica y el desarrollo cognitivo, al respecto, explica:

"Nuestra ciencia de trabajo es la Educología. Es una nueva ciencia que fusiona la cultura ecológica con el desarrollo cognitivo del individuo. La Educología apuesta por la creación y gestión de un aula ecológica en la escuela utilizando el huerto ecológico como herramienta pedagógica de primer orden para facilitar al escolar la comprensión de las interrelaciones de los seres humanos entre sí y con la Naturaleza de una forma amena, dinámica y divertida. Desde la Educología se promueve la adquisición de hábitos alimenticios saludables, equilibrados y sostenibles, a través del conocimiento de los productos que consumimos, su origen, sus posibilidades culinarias También se potencia la comprensión de conceptos como la biodiversidad, la ecología, el desarrollo sostenible, el respeto medioambiental y la gestión de los residuos y el reciclaje; al tiempo que se fomentan valores tan valiosos como el trabajo en equipo, la igualdad, la responsabilidad, el compromiso, el respeto, la constancia, la valoración del propio esfuerzo, Y además, se acerca a los alumnos a la cultura rural y agrícola, la cual es tan antigua como el ser humano que ahora vuelve a resurgir llena de nuevos matices (educativa e incluso terapéutica".

Definición con la que compartimos plenamente, por cuanto la educología estudia, investiga y explica el hecho, proceso y fenómeno educativo desde un visón holística, integrando el conocimiento ecológico con el conocimiento cognitivo que le da una connotación de una educación sostenible y sustentable.

Desde este punto de vista, la educología que estudia al hecho o fenómeno educativo vinculado y articulado a la ciencia ecológica que busca y promueve la conservación del medio ambiente, por cuanto; no puede haber una educación de calidad sin el cuidado del medio ambiente para no perjudicar a las futuras generaciones. 
También la educología se preocupa por la sostenibilidad de la educación. La educología como ciencia o epistemología promueve la gestión sostenible y sustentable de la educación para formar conciencia ecológica para no perjudicar a las futuras generaciones. La educología, como una nueva ciencia o epistemología educativa, integra holísticamente el nuevo concepto de educación. La educología integra la educación del ser humano con la naturaleza.

Calixto Flores, R. (2012) en su artículo titulado: Investigación en educación ambiental, al referirse a la necesidad de una educación ambiental o ecológica, sostiene:

Los problemas ambientales detonan el surgimiento de la era, ya que el objeto de estudio de ésta es el medio ambiente. La educación ambiental se propone, a través del desarrollo de diversas estrategias pedagógicas, contribuir a la formación de una conciencia sobre la responsabilidad del género humano en la continuidad de las distintas formas de vida en el planeta, así como la formación de sujetos críticos y participativos ante los problemas ambientales".

Desde este punto de vista tenemos que redefinir el concepto de Educación.

Desde la perspectiva Educológica, la educación es un proceso continuo de formación de la conciencia social histórica para transformar la sociedad. La educación debe ser un proceso holístico para generar un desarrollo sostenible y sustentable, considerando como eje principal al ser humano y el medio ambiente o la naturaleza en que vivimos. La educación es un proceso formativo de la articulación, de lo cognitivo con lo ecológico para no perjudicar a las futuras generaciones.

En el contexto escolar interactúan los actores educativos que están conformados por los actores siguientes: El educólogo y el educando.

\section{Visión holística de la educología}

La educología como una emergente ciencia o epistemología de la educación, asume una visión holística en que vivimos, por cuanto enlaza el aspecto cognitivo con lo ecológico. La educología sostiene que la educación debe ser sostenible y sustentable, lo cual significa que, la educación debe realizarse teniendo en cuenta como eje principal al ser humano y a la madre naturaleza en cuyo contexto nos desarrollamos. 


\section{Cosmovisión holística de la educología}

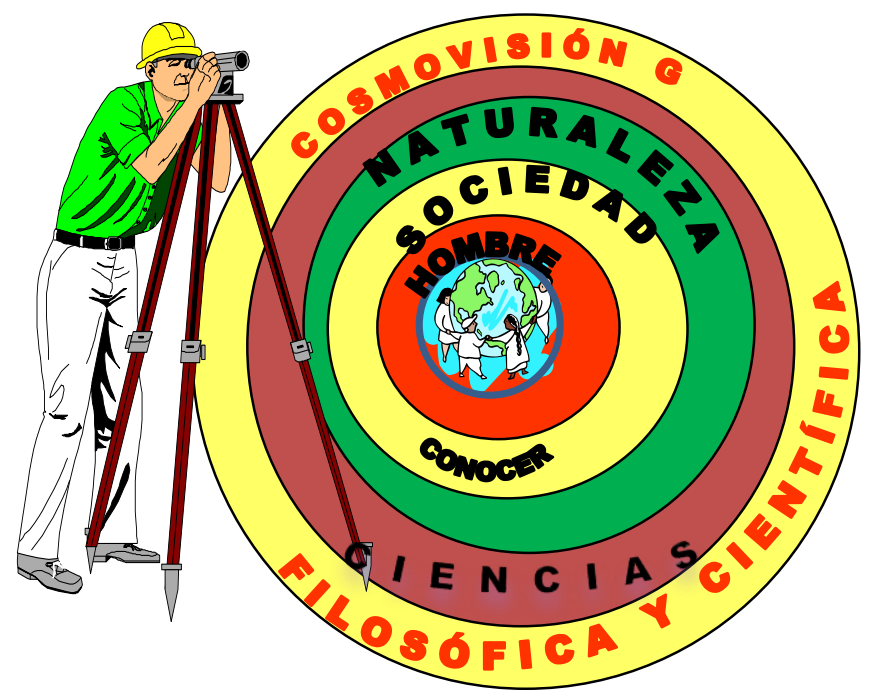

Franco Gómez, M del C. (2017) en su artículo titulado: Visión holística de la educación ambiental y el desarrollo sostenible. buenas prácticas en la universidad metropolitana del ecuador, sostiene que la articulación entre la educación y desarrollo sostenible, es vital, al respecto, explica:

La educación es determinante en la implementación de este nuevo enfoque de desarrollo; se encarga de incitar el establecimiento de nuevos y positivos modos de relación del ser humano con su medio ambiente, abarcando su diversidad y que está dada en las áreas sociales, naturales, tecnológicas, económicas o políticas. Debe establecerse como punto de partida en la necesaria incidencia sobre los diferentes actores sociales, para de esta forma potenciar la adquisición de conocimientos, valores y estilos de conducta prósperos para el medio ambiente.

La educología, como una meta teoría de la educación, nos señala que el nuevo concepto de educación debe estar articulada el aspecto cognitivo con el aspecto ecológico. El educólogo debe formar conciencia ecológica.

\section{¿Qué es el educólogo?}

El educólogo es el profesional de la educación, dotado de una alta teoría epistemológica llamado Educología. 
El educólogo como uno de los actores educativos de la educación, debe tener una alta preparación con dominio filosófico, epistemológico, psicológico, sociológico, antropológico y otras ciencias afines al que hacer educativo.

Sobre todo, deber no solo una visión holística, ecuménica, sino debe tener una cosmovisión, concepción y un pensamiento complejo transdisciplinar con una alta conciencia social histórica y ecológica.

\section{¿Qué es la educogenia?}

La Educogenia es la educación que logramos construir al tener nuestro primer embrión de vida, también es aflorar todo el potencial que emerge desde nosotros desde nuestra concepción hasta el día de nuestra muerte. Es todo aquello que aflora desde nuestra identidad más íntima.

\section{DISCUSIÓN DE RESULTADOS}

Para nuestro punto de vista la Educación no es Ciencia, por cuanto; no tiene su propio objeto o campo de estudio, es decir; no tiene su parcela del saber. Además, la Educación constituye un hecho, proceso y fenómeno que constituye un campo de estudio de otra ciencia, en este caso. Esa ciencia emergente es la Educología, por cuanto; como ciencia, estudia al hecho o fenómeno educativo.

Todo saber humano para configurase como ciencia debe tener una estructura sólida que no genere dudas para constituye como una ciencia.

Los que defienden a la educación como ciencia, no presenta con claridad su campo u objeto de estudio.

Igualmente, los defensores de la Pedagógica como ciencia no presentan con claridad su objeto de estudio.

Tanto la educación y la pedagogía se vienen disputando al hecho o fenómeno como su campo de estudio.

Frente a esta divergencia, surgen o emergente una nueva ciencia o epistemología de la educación llamado Educología.

La educología como ciencia tiene una estructura sólida que tiene su objeto de estudio constituye el hecho o fenómeno educativo.

La educología también tiene sus propios métodos, leyes, teorías, categorías, ramas y disciplinas que le permite tener un propio Estatuto para configurarse como ciencia. 


\section{CONCLUSIONES}

Como resultado del análisis bibliográfico llegamos a la conclusión que en la actualidad persiste una lucha epistemológica por configurarse como ciencia tanto la Educación y la Pedagogía. Como conclusión tenemos lo siguiente:

1. Existen divergencias y controversias entre los que defienden a la Educación y Pedagogía, una lucha epistemológica que continua vigente en la actualidad.

2. La lucha epistemológica por llamarse ciencia tanto la Educación y la Pedagogía no acaba, continua vigente.

3. Hay muchos destacados autores que defienden a la Educación como ciencia, pero no presenta con claridad, pero no presenta cuál es su objeto o campo de estudio.

4. También hay otros estudiosos que afirman en decir que la Pedagogía constituye una ciencia. De igual forma los que defienden la tesis de que la Pedagogía como ciencia no presente con claridad sus argumentos sobre el objetivo o campo de estudio que le da personalidad propia para llamarse ciencia.

5. La Educología como una nueva ciencia tiene su propio campo u objeto de estudio. El objeto o campo de estudio de la Educología está conformado por los hechos y fenómenos educativos articulados dialécticamente a la conciencia ecológica. Es la nueva ciencia que fusiona el desarrollo cognitivo con la cultura ecológica del ser la persona como ser social.

\section{REFERENCIAS BIBLIOGRÁFICA:}

Abreu-Valdivia, O. y otros (2021) La pedagogía como ciencia: su objeto de estudio, categorías, leyes y principios. Inf. tecnol. vol.32 no.3 La Serena jun. 2021.

Arias Monge, M. y Navarro Camacho, M (2017) Epistemología, Ciencia y Educación Científica: premisas, cuestionamientos y reflexiones para pensar la cultura científica. Revista Electrónica “Actualidades Investigativas en Educación”.

Asensi-Artiga, V. y Parra-Pujante, A. (2002) El método científico y la nueva filosofía de la ciencia. Anales de documentación, n. ${ }^{o}$ 5, págs. 9-19, Universidad de Murcia.

Bujardón Mendoza, A. (2008) eflexiones epistemológicas acerca de la educación en valores humanos. Rev Hum Med v.8 n.2-3 Ciudad de Camaguey, mayo-dic.

Calixto Flores, R. (2012) Investigación en educación ambiental. RIMIE, VOL. 17, núm. 55. 
Díaz Genis, A. y Camejo, M. (2014) Epistemología y educación Articulaciones y convergencias. Rev. Interdisciplinario Universidad de la República de Uruguay.

Di Claudo, M. V. (2013) La ciencia pedagógica: construcciones, disputas, desafíos. Sophia 14: 2013. Universidad Politécnica Salesiana del Ecuador.

Franco Gómez, M. y otros (2017). Visión holística de la educación ambiental y el desarrollo sostenible. Buenas prácticas en la universidad Metropolitana del Ecuador. Revista Conrado,13(1-Ext),138-141.

Gonçalves da Cunha, S.

Gonzales, Alvarado, F. (2008) La pedagogía como disciplina: Aproximaciones teóricas y reflexiones desde la práctica. Rev. Pensamiento Pedagógico. Universidad Nacional de Costa Rica.

Guichot Reina, V. (2006) Historia de la Educación: reflexiones sobre su objeto, ubicación epistemológica, devenir histórico y tendencias actuales. Revista Latinoamericana de Estudios Educativos (Colombia), vol. 2, núm. 1, enero-junio, 2006, pp. 11- 51 Universidad de Caldas Manizales, Colombia.

Guevara Gálvez, B. (2015) Educología, epistemología de la educación. Lima, Ediciones Pensamiento y Acción.

Hernández Huerta, J. L. y otros (2014)

Lizarazo, J. S. (2018) Economía Ecológica y la construcción epistemológica de una ciencia revolucionaria para la sostenibilidad y la transformación del mundo. Gestión y Ambiente 21(supl. 1), 13-34, 2018

López Noguero, F. (2002) El análisis de contenido como método de investigación. Revista de Educación, 4 167-179. Universidad de Huelva.

López Rupérez, F. (1990) Historia y Epistemología de la Ciencias. Instituto Español de París.

Loreto Mora Olate, M. (2020) Educación como disciplina y como objeto de estudio: aportes para un debate. Desde el Sur vol.12 no.1 Lima ene. jun.

Maranto Rivera, M. y María Eugenia González Fernández, M. E. ¿Qué es la Ciencia? http://www.uaeh.edu.mx/virtual.

Méndez, E. (2000) El desarrollo de la ciencia. Un enfoque epistemológico. Rev. Espacio Abierto, vol. 9, núm. 4, octubre-diciembre, pp. 505-534 Universidad del Zulia Maracaibo, Venezuela. 
Mateos Montero, J. (2014) Pedagogía: ¿arte o ciencia? Un viejo debate inacabado. España Colección Ágora, n. 2.

Meza Cascante, L. G. (s/f) La Educación como Pedagogía o como Ciencia de la Educación.file:///C:/Users/HP/Downloads/6739.

Moreno Castillo, R. (2009) ¿Es la pedagogía una ciencia? Foro de Educación, n. ${ }^{\circ}$ 11, pp. 67-83.

Oviedo, P. P. J. (1979) Educación Y Ciencia. El Basilisco, número 7, mayo-junio 1979, www.fgbueno.es

Reyes Solís, D. (2006) Problemas epistemológicos de las ciencias humanas y de la educación alteridad. Revista de Educación, vol. 1, núm. 1, 2006, pp. 19-28 Universidad Politécnica Salesiana Cuenca, Ecuador.

Sáez Alonso, R. (2016) Teoría de la educación: conocimiento de la educación, investigación, disciplina académica. Revista Virtual Redipe: Año 5 Volumen 8

Vega Román, E.A. (2018) ¿Pedagogía o ciencias de la educación? una lucha epistemológica. Revista Boletín REDIPE 7 (9): 56 - 62 - s e p t i e m b r e.

Vicente, M.E. (2016) Ciencias de la

Educación: nuevas definiciones profesionales desde la historia reciente.

Argentina, Revista Trabajo y Sociedad, núm. 27, pp. 155.

Viveros Viveros, W. S. (2019) La educación científica y la filosofía de la ciencia: una

relación necesaria como propuesta académica en el currículo. Revista Conrado, 15 (70), 384-391.

Zambrano Leal, A. (2002) Las ciencias de la educación y didáctica: hermenéutica de una relación culturalmente específica. Rev. Educere, vol. 10, núm. 35, octubrediciembre, pp. 593-599 Universidad de los Andes Mérida, Venezuela. 